\title{
Metastasizing Chondroblastoma
}

National Cancer Institute

\section{Source}

National Cancer Institute. Metastasizing Chondroblastoma. NCI Thesaurus. Code C66799.

A rare chondroblastoma that has metastasized to another anatomic site. 